\title{
Numerical Analysis of a Non-Newtonian Fluid in Agitated Vessel with PBT-Rushton Combined Impeller
}

\author{
U. I. Mohd Ali, A. Zamiri, S. Y. Lee, J. T. Chung \\ Mechanical Engineering Department/Korea University \\ Anam-ro, Seoul, Korea \\ ummualyahya@korea.ac.kr; ali.en.zamiri@gmail.com; seungyobp@korea.ac.kr; jchung@korea.ac.kr
}

\section{Extended Abstract}

Mixing operation in mechanically agitated vessel is vital in industrial processes such as pharmaceutical, food processing as well as paint production industry. Study of mixing in Newtonian and non-Newtonian fluid has been explored extensively with myriad of impeller geometries. In contrast to Newtonian fluid, mixing process in non-Newtonian fluid is very complicated and it involves complex flow condition inside the agitated system. Therefore, a detailed investigation on hydrodynamic behaviour in agitated vessel is crucial to improve mixing efficiency for enhancing quality of product.

Among the numerous impeller types studied in Newtonian fluid, investigation on high-speed impellers such as pitched-blade turbine (PBT) and Rushton impellers cover large portion of the studies which can be concluded that PBT is suitable for flow-sensitive operation while Rushton is favourable for dispersion. Geometry of PBT does not give significant effect on power number except for impeller-to-tank diameter ratio, while for Rushton impeller, power number is highly dependent on impeller geometry [1]. A numerical study had been employed on fluid mixing with a novel impeller design, which combines PBT and Rushton impeller. The PBT-Rushton combined impeller had shown the significant improvement in terms of velocity field in addition to circulation size of primary and secondary loops [2].

Due to complex rheological behaviour of non-Newtonian fluid, discharge flow generated by impeller can be completely different from Newtonian fluid. According to Kazemzadeh et al. [3], PBT impeller discharges radial flow in the yield-pseudoplastic fluid, instead of discharging in axial direction, which was also reported by Rudolph et al. [4]. Furthermore, axial velocity magnitude attained by PBT impeller was higher than Rushton impellers. This is the importance of PBT blade in the PBT-Rushton combined impeller. On the other hand, they observed that Rushton impeller produce more stable flow pattern than PBT. This shows that the existence of Rushton blade is needed in PBT-Rushton combined impeller to promote uniformity of discharge flow for more efficient mixing system.

In this present work, the main objective is to investigate the performance of the PBT-Rushton combined impeller in xanthan gum solution as a non-Newtonian fluid. Therefore, 3-D incompressible Reynolds-Averaged Navier-Stokes (RANS) equation was numerically modelled in commercial software, ANSYS CFX V18. The model is made up of two main parts consisted of stationary and rotating domains. Performance of PBT-Rushton combined impeller is compared with high-speed impellers: Scaba, PBT, Rushton and PBT-Rushton with curved blade impeller. Additionally, PBTRushton with curved blade impeller is simulated in view of the fact that Rushton with curved blade requires less power consumption than standard Rushton blade.

CFD results are validated by comparing with experimental data reported by Venneker et al. [5] in terms of velocity field. Performance of impellers is evaluated by flow pattern, flow number, power number and turbulent characteristics in agitated vessel generated by the impellers. Pressure is evenly distributed at the edge of PBT-Rushton combined impeller blade while pressure is concentrated the centre of curved blade of Scaba and PBT-Rushton with curved blade. Reduction in pressure gradient at near-impeller region of curved blade leads to decrement in power consumption. A pair of trailing vortices are created behind the blade of Rushton impeller while a single vortex is generated by the blade of PBT impeller, which was also observed by Ranade et al [6]. In the case of PBT-Rushton impeller, similar vortices can be seen behind both PBT and Rushton blades that can provide high turbulence level near the impeller. Furthermore, the trailing vortices formed behind the PBT-Rushton with curved blade are longer than those of Scaba impeller.

\section{References}


[1] D. Chapple, S. M. Kresta, A. Wall, and A. Afacan, "The Effect of Impeller and Tank Geometry on Power Number for a Pitched Blade Turbine," Chem. Eng. Res. Des., vol. 80, no. 4, pp. 364-372, 2002.

[2] U. I. Mohd Ali, A. Zamiri, and J. T. Chung, "Numerical Analysis of Axial-Radial Combined Impeller Design in Unbaffled Agitated Vessel," Int. Conf. of Comp. Heat, Mass and Momentum Trans, Korea, 2017.

[3] A. Kazemzadeh, F. Ein-Mozaffari, A. Lohi, and L. Pakzad, "A New Perspective in the Evaluation of the Mixing of Biopolymer Solutions with Different Coaxial Mixers Comprising of Two Dispersing Impellers and a Wall Scraping Anchor," Chem. Eng. Res. Des., vol. 114, pp. 202-219, 2016.

[4] L. Rudolph, M. Schaefer, and M. Kraume, "Power Consumption and Blend Time of Co-Axial Tank Mixing Systems in Non-Newtonian Fluids," $13^{\text {th }}$ Eur. Conf. on Mixing, pp. 14-17, 2009.

[5] B. C. H. Venneker, J. J. Derksen, and H. E. A. Van den Akker, "Turbulent Flow of Shear-Thinning in Stirred TanksThe Effects of Reynolds Number and Flow Index," Chem. Eng. Res. Des., vol. 88, no. 7, pp. 827-843, 2010.

[6] V. V. Ranade, Y. Tayalia, and H. Krishnan, "CFD Predictions of Flow Near Impeller Blades in Baffled Stirred Vessels: Assessment of Computational Snapshot Approach,” Chem. Eng. Commun., vol. 189, pp. 895-922, 2002. 